\title{
REVIEW
}

\section{Skin innate immune response to flaviviral infection}

\author{
Magali Garcia $^{1,2}$, Michel Wehbe ${ }^{1}$, Nicolas Lévêque ${ }^{1,2}$, Charles Bodet ${ }^{1}$ \\ ${ }^{1}$ Laboratoire inflammation tissus épithéliaux et cytokines EA 4331, université de Poitiers, Poitiers, France \\ ${ }^{2}$ Laboratoire de virologie et mycobactériologie, CHU de Poitiers, Poitiers, France
}

Correspondence: Charles Bodet, LITEC EA 4331, université de Poitiers, B36, 1, rue Georges-Bonnet, TSA 51106, 86073 Poitiers cedex 9, France. <charles.bodet@univ-poitiers.fr>

Accepted for publication May 18, 2017

To cite this article: Garcia M, Wehbe M, Lévêque N, Bodet C. Skin innate immune response to flaviviral infection. Eur. Cytokine Netw. 2017; 28(2): 41-51 doi: $10.1684 /$ ecn. 2017.0394

\begin{abstract}
Skin is a complex organ and the largest interface of the human body exposed to numerous stress and pathogens. Skin is composed of different cell types that together perform essential functions such as pathogen sensing, barrier maintenance and immunity, at once providing the first line of defense against microbial infections and ensuring skin homeostasis. Being inoculated directly through the epidermis and the dermis during a vector blood meal, emerging Dengue, Zika and West Nile mosquito-borne viruses lead to the initiation of the innate immune response in resident skin cells and to the activation of dendritic cells, which migrate to the draining lymph node to elicit an adaptive response. This literature review aims to describe the inflammatory response and the innate immune signalization pathways involved in human skin cells during Dengue, Zika and West Nile virus infections.
\end{abstract}

Key words: flavivirus, inflammation, signalling pathway, RIG-I, TLR3, MDA5

Flaviviruses are $\sim 11 \mathrm{~kb}$, positive-sense, single-stranded, RNA-enveloped viruses transmitted by an arthropod vector during its blood meal, and are therefore classified as "arboviruses" (arthropod-borne viruses).

The Flavivirus genus contains more than 70 viruses among which Dengue (DENV), Zika (ZIKV) and West Nile viruses (WNV) are emergent, posing a significant worldwide threat as illustrated by recent epidemics of ZIKV and WNV on the American continent [1, 2]. Following their inoculation into the dermis and the epidermis, virus replication can be detected at the site of inoculation from the first day of infection [3, 4]. Skin also represents the first line of defense against these pathogens as it generates early immune response to flaviviral infection [5]. Human skin is a complex organ organized in three successive layers: the outer multilayered epidermis, the dermis and the inner hypodermis [6]. The epidermis is composed mainly of keratinocytes, which represent more than $90 \%$ of the cells, melanocytes, and Langerhans cells (LCs), a specialized type of dendritic cell (DC) that constantly probes for antigen in the most exposed, superficial layer of the skin [7]. After being activated by an encounter with an antigen, LCs can migrate to the draining lymph node, leading to T-cell priming [8]. The dermis is composed of numerous distinct cells such as fibroblasts, endothelial cells or more specialized immune cells such as mast cells, DCs, T lymphocytes and macrophages [6], which can play a role in the immune skin barrier. This literature review aims to describe the skin's inflammatory response and the innate immune signalization pathways involved in human skin cells during DENV, ZIKV and WNV infections.

\section{INTERACTIONS BETWEEN DENV, ZIKV AND WNV AND SKIN CELLS}

\section{Skin cell permissivity to DENV}

Dengue is the second tropical infectious disease in the world with up to $2 / 3$ of the world population exposed [9]. There exist 4 serotypes of DENV (DENV1 to DENV4), each one leading indistinctly to pathologies ranging from mild febrile disease to life-threatening haemorrhagic shock [10]. Several studies have demonstrated the ability of DENV to replicate in cadaveric [11] as well as in non-cadaveric human skin explants [12], particularly in immature DCs. Murine models of skin infection with DENV have revealed that DCs are infected in two stages: an initial infection of resident dermal DCs within 12-24 h after virus inoculation and a delayed infection within $48 \mathrm{~h}$ after virus inoculation of DCs derived from monocytes recruited to the inflamed dermis [13]. Moreover, subcutaneous injection of DENV in mice or monkeys results in highly active migration of DCs within $24 \mathrm{~h}$ from the skin to the local lymph nodes. Dermal DC migration was shown to be more dependent on tumor necrosis factor (TNF) $\alpha$ than on interleukin (IL) $1 \beta$ secretion $[14,15]$. Finally, a healthy volunteer woman who received a tetravalent, live attenuated DENV deltoid vaccination presented with skin macules and papules at the twelfth day post intramuscular inoculation, which disappeared after 6 days [11]. During the cutaneous rash, histological and immunohistochemical analysis of a skin biopsy showed a mild superficial dermal, perivascular lymphocytic infiltrate and the expression of DENV antigens in LCs [11]. 


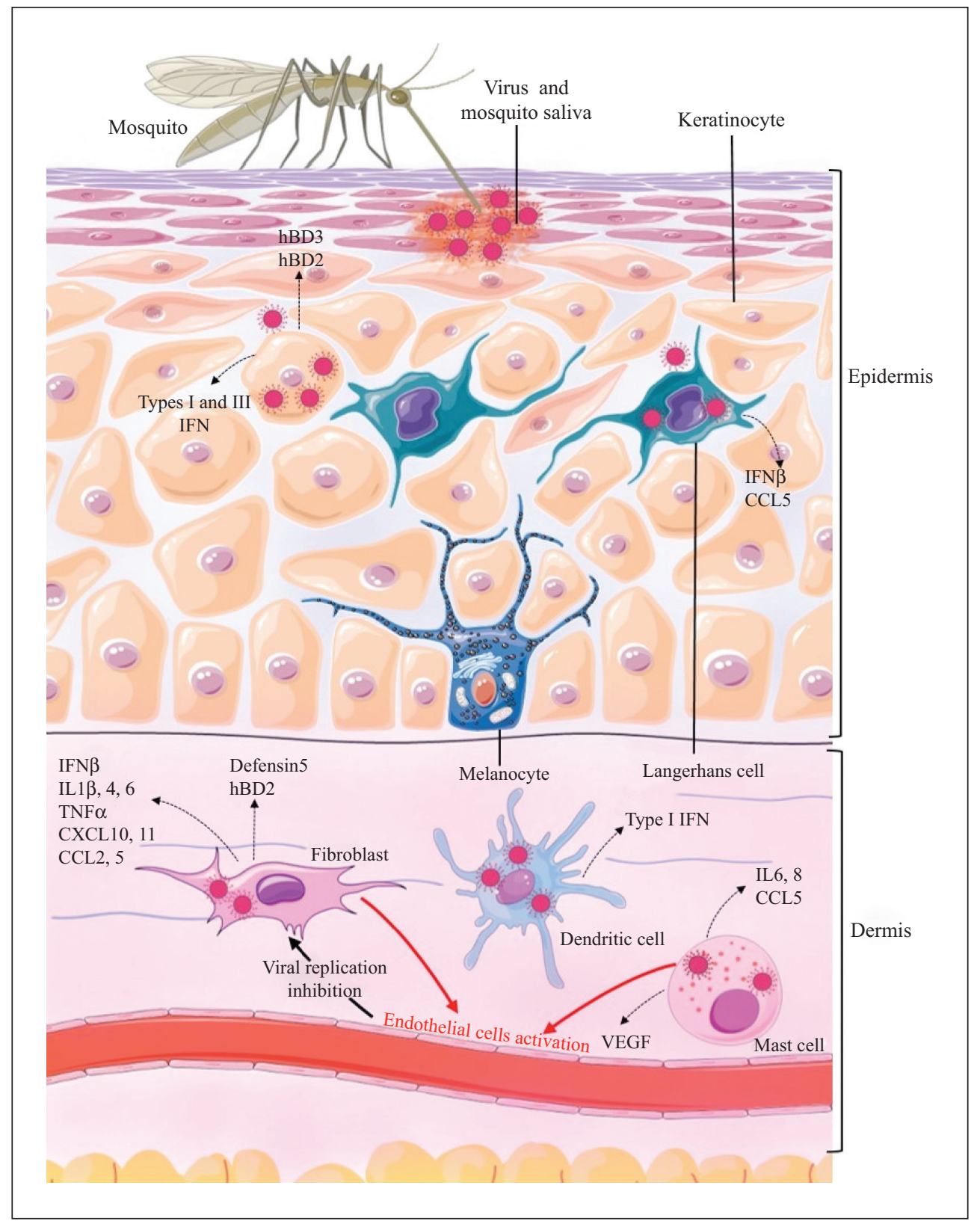

Figure 1

Schematic first steps of Dengue (DENV), West Nile (WNV) and Zika (ZIKV) viruses inoculation through the human skin, replication and induction of inflammatory response. Following the probe of an infected mosquito, the virus can replicate in keratinocytes (DENV, WNV and ZIKV), fibroblasts (DENV, WNV and ZIKV), skin dendritic cells (DENV and WNV), Langerhans cells, dermal dendritic cells and mast cells (DENV) inducing an antiviral response mainly constituted of type I and III IFNs, IL4, IL1 $\beta$, IL6, CCL5, IL8, CXCL10, CXCL11 and antimicrobial peptides such as defensin 5, hBD2 and 3. Mast cell and fibroblast infection by DENV can lead to endothelial cell activation and proliferation via secretion of VEGF. In return, endothelial cells can secrete soluble mediators able to inhibit DENV replication.

Human primary keratinocytes from neonatal foreskins were also shown to be permissive to DENV2 infection, which induced not only an early and strong interferon (IFN) $\beta$ mRNA expression but also a weaker and fleeting IFN $\gamma$ and antimicrobial peptide (AMP) expression [16]. AMPs were induced from $6 \mathrm{~h}$ of infection but with differences in kinetics: human $\beta$ Defensin (hBD)3 mRNA expression rapidly increased, followed by a progressive decrease at $48 \mathrm{~h}$ post-infection (p.i.); hBD2 was mainly expressed after $48 \mathrm{~h}$ of infection, while RNase 7, another AMP expressed in human skin, was poorly induced (figure 1, [16]).

In the dermis, several studies have assessed the permissiveness of human dermal fibroblasts (HDFs) to DENV in vitro [17-19], which has been associated with induction of a wide range of inflammatory markers such as
IFN $\beta$, TNF $\alpha$, IL4, IL1 $\beta$, IL6, chemokine (C-C motif) ligand (CCL)2 (also known as monocyte chemoattractant protein $1 \mathrm{MCP} 1$ ), CCL5, defensin 5 and hBD2 mRNA expression and TNF $\alpha$, defensin 5, hBD2, CCL5, granulocyte macrophage colony-stimulating factor (GM-CSF) and IFN $\beta$ production $[17,19]$. As fibroblasts are near endothelial cells in the dermis, Bustos-Arriaga et al. investigated whether DENV-infected HDFs could activate human dermal microvascular endothelial cells (HDMECs) using a co-culture model. They showed that the soluble mediators of innate immunity secreted by the co-cultures of DENVinfected HDFs and HDMECs were sufficient to reduce viral replication, activate HDMECs and induce leukocyte migration through HDMEC monolayers [18]. Moreover, they demonstrated that innate immunity might be differentially activated according to DENV serotypes since 
pro-inflammatory mediator secretion patterns in HDF and HDMEC co-cultures were specific to DENV2 or DENV4 infection [18]. Overall, these results highlight the crosstalk between skin-resident HDFs and HDMECs, which can contribute to the creation of an immune-activated microenvironment and antiviral state.

Previous studies have demonstrated a dual role of mast cells in DENV pathophysiology by using mast cell-deficient mice, a role that is either protective by reducing vascular permeability or aggravative by enhancing DENV infectivity and macrophage skin infiltrates at the inoculation site compared to wild-type mice [20, 21]. Troupin et al. recently confirmed their crucial role in DENV replication and systemic propagation [22]. They demonstrated that human skin mast cells were permissive to DENV following exposure of samples of human skin tissue to infected mosquitoes [22]. DENV infection of skin mast cells resulted in upregulation of specific immune-related genes and secreted levels of CCL5, IL8 and IL6, as well as endothelial growth factors, particularly vascular endothelial growth factor (VEGF) [22]. Moreover, using transwell assays, the ability of DENV-infected human skin mast cells to signal neighbouring HDMECs for activation (increased expression of intercellular adhesion molecule [ICAM] and vascular cell adhesion molecule [VCAM]) and proliferation was reported (figure 1; [22]). Interestingly, infectious DENV was found to localize in the mast cell cytoplasmic granules and, also, after degranulation, in extracellular granules, thereby contributing to the spread of viruses within the host body from the inoculation site [22].

\section{Skin cell permissivity to $W N V$}

WNV is a neurotropic flavivirus endemic in the United States of America since 1999 and in Europe [23, 24]. Usually asymptomatic, infection by this virus can cause mild febrile disease and, rarely, an encephalitic presentation [25]. As its transmission occurs after a skin probe of a Culex or an Aedes mosquito, its ability to infect skin cells has been investigated. After subcutaneous infection of mice in both rear footpads with $10^{5}$ plaque-forming units, Lim et al. detected WNV-positive cells by immunohistochemistry, mainly in focal areas of the epidermis and in epithelial cells of adnexal glands associated with hair follicles [26]. The infected epidermal cells were identified as keratinocytes by double labelling for WNV antigens and keratin 10 [26]. Moreover, studies have demonstrated the ability of WNV to persist in the skin from 1 to 4 months post-inoculation in sparrows [27] and mice [28], respectively. In humans, high titres of WNV have been produced in primary skin cultures and keratinocytes for at least 6 days in vitro [26]. Human skin fibroblasts are also permissive to WNV in which viral replication has induced TNF $\alpha$ and IFN $\beta$ secretion [29]. However, Hoover and Fredericksen suggested that fibroblasts did not play a major role in vivo during WNV infection because of the production of low-infectivity particles [30].

In addition, several studies have demonstrated that human monocyte-derived DCs were permissive to WNV [31-35] and secreted high amounts of type I IFN but low levels of pro-inflammatory cytokines such as IL12, IL23, IL18 or IL10 in response to infection [36]. In the skin, or after migration to cutaneous lymph nodes, infected DCs then interact with natural killer T cells (NKTs), an innate effector cell type important for control of pathogen infections [37]. Nonetheless, WNV-infected human DCs are not able to activate the pro-inflammatory functions of invariant NKTs, a type of NKT specifically contributing to antiviral response [36, 38].

Interestingly, type I IFN has demonstrated a paradoxical role during WNV infection. On the one hand, IFN signalling has been described as decreased in DCs from aged donors infected with WNV, a phenomenon that could partially explain the more severe WNV infections observed in older people [32]. On the other hand, mice not expressing the AXL receptor tyrosine kinase, which is induced in response to type I IFN and exerts a negative feedback loop on IFN production, showed enhanced susceptibility to WNV, with increased type I IFN response associated with diminished DC maturation, reduced production of IL1 $\beta$, and defective antiviral $\mathrm{T}$ cell immunity [39]. Moreover, in BALB/C mouse, cutaneous WNV infection resulted in upregulation of major histocompatibility complexes, (MHC)II and ICAM-1. Expression of the costimulatory molecule CD80 (B7-1) in epidermal DCs and LCs was also increased and their migration from the epidermis to the local draining lymph nodes, where they are responsible for the activation of $\mathrm{T}$ cells in primary immune response, was induced $[40,41]$. This migration was dependent on IL1 $\beta$ secretion $[40,42,43]$ whereas TNF $\alpha$ secretion seemed not essential [43].

\section{Skin cell permissivity to ZIKV}

ZIKV was recently involved in the hugest arbovirus epidemic that the world has known, illustrating its ability to be sexually transmitted and to cause foetal abnormalities when contracted during pregnancy [44]. There exist two major lineages of ZIKV, one Asian and the other African [45]. Hamel et al. demonstrated that human dermal fibroblasts, epidermal keratinocytes and immature dendritic cells were permissive to ZIKV French Polynesia strain (Asian strain), whereas Bowen et al. demonstrated that human DCs were permissive to both African and Asian isolates $[46,47]$. Detection of viral antigens could be observed in primary human epidermal keratinocytes as soon as $24 \mathrm{~h}$ p.i., reaching $100 \%$ of cells after $72 \mathrm{~h}$ of infection, while viral RNA was detected as soon as $6 \mathrm{~h}$ p.i. in human primary epidermal keratinocytes and fibroblasts [46]. After infection of human skin explants with ZIKV, viral genome copy number increased gradually until maximal levels were obtained at 5 days p.i. and histological changes such as cytoplasmic vacuolation, apparition of pyknotic nuclei in keratinocytes of the stratum granulosum and sporadic oedema in the sub-corneal layer were observed in infected skin explants [46]. Otherwise, ZIKV induces an innate immune response in primary human skin fibroblasts characterized by enhanced expression of two CXCR3 ligands: chemokine (C-X-C motif) ligand CXCL10 and CXCL11, attracting leukocytes to the infectious site and presenting antimicrobial activity (figure 1 ; [48]), and of CCL5, an inflammatory antiviral chemokine. In skin fibroblasts, pretreatment with increasing concentrations of INF $\alpha, \beta$, and $\gamma$ has led to reduced ZIKV replication and viral excretion in cell culture supernatant [46]. In contrast, human DCs infected by ZIKV have led to an induction of IFN $\beta$ mRNA expression without either increased protein or restricted virus replication [47]. 
Hence, it is well established that various resident and migratory skin cells, such as keratinocytes, fibroblasts, mast cells or DCs, are involved in inflammatory response to flaviviral infection. In addition, even if their role in flaviviral infection still needs to be demonstrated, recent works have suggested that platelets should be considered as immune cells in the skin as regard their ability to recognize pathogens, to release AMPs, chemokines and cytokines, to recruit leucocytes and to induce neutrophil phagocytosis [49]. Finally, melanocytes have been suspected of playing a role in skin innate immune response contributing to the phagocytosis of pathogens, presenting antigens to competent immune cells or producing cytokines such as IL1 $\beta$, IL6 and TNF $\alpha$ as well as chemokines [50].

\section{Flavivirus sensing}

After viral inoculation by the mosquito in the skin, the virus bounds to the host's cell receptor, which is probably highly conserved as the natural cycle transmission alternatively involves arthropods and vertebrates [51]. This currently unknown receptor has been suspected to be closely linked with the dendritic cell-specific ICAMgrabbing non-integrin (DC-SIGN), T-cell immunoglobulin and mucin domain (TIM) and TYRO3, AXL and the MER (TAM) proteins involved in flavivirus attachment at the cell surface $[46,52]$. Thereafter, the virus is internalized by a receptor-mediated endocytosis enabling release of the nucleocapsid in the cell cytoplasm. The positive singlestranded (ss) genomic RNA is translated into a polyprotein secondary cleaved by cellular and viral proteases into three structural proteins (capsid C, pre-membrane/membrane $\mathrm{prM} / \mathrm{M}$ and envelope E) and seven non-structural (NS1, NS2A, NS2B, NS3, NS4A, NS4B and NS5) proteins that are required for virus replication. ssRNA can also be transcribed in order to obtain a complementary negative ssRNA, thereby constituting a double-stranded replicative form (ds-RF) [51]. The negative ssRNA synthesized will be used in turn as a template for simultaneous synthesis of multiple positive ssRNA, thereby constituting partial double-stranded (ds) replicative intermediates (RI), which can be sensed by the host cell innate immune receptors $[51,53,54]$. Finally, after virion assembly through the endoplasmic reticulum, viral particles are released from the cell by exocytosis [55]. Several studies have demonstrated that the viral replication process is vital to the induction of increased expression of RIG-I-like receptors (RLRs), an inflammatory response or migration of DCs from the skin to the draining lymph node $[15,41,56]$. During viral infection, cells must quickly induce an innate antiviral response in order to attempt to control viral replication, providing time for the adaptive arm of the immune system to establish an effective response. Hence, all the cell types found in the skin, including keratinocytes [57], LCs [58], melanocytes [59], macrophages, DCs, T and B cells, mast cells [60], endothelial cells [61, 62], fibroblasts and adipocytes express the receptors of innate immune system called pattern-recognition receptors (PRRs), which recognize conserved pathogen-associated molecular patterns (PAMPs), exclusively expressed by microorganisms and not by host cells, and danger-associated molecular patterns (DAMPs), which are danger signals expressed by stressed host cells, enabling host defenses to sense and elicit immune responses against pathogens [63].
PRRs are subdivided into 3 kinds of receptors: Toll-like receptors (TLRs) located on the cell membrane or in endosomes, retinoid-inducible helicases receptors (RLRs) and Nod-like receptors (NLRs), which are both cytoplasmic. Flaviviral dsRNA and ssRNA are PAMPs that can respectively be sensed by TLR3 and TLR7 (figure 2; [64]). TLR activation triggers downstream signalling pathways that activate innate immune defenses. TLR3 recruits the adaptor molecule TRIF (TIR domain-containing adaptor inducing IFN $\beta$ ) and activates the transcription factors interferon regulatory factor (IRF)3, IRF7 and nuclear factor $-\kappa \mathrm{B}(\mathrm{NF}-\kappa \mathrm{B})$ to trigger the production of type I and III IFNs (IFN $\alpha, \beta, \lambda$ ), pro-inflammatory cytokines, chemokines and interferon-stimulated genes (ISGs) [65]. TLR7 recruits the adaptor molecule myeloid differentiation 88 (MyD88) and activates transcription factors IRF7 and NF- $\mathrm{kB}$ leading to the expression of type I and III IFNs and pro-inflammatory cytokines [65].

RIG-I (retinoic acid-inducible gene 1), MDA5 (melanoma differentiation antigen 5) and LGP2 (laboratory of genetics and physiology 2) belong to RLRs cytoplasmic RNA helicase family, which plays a critical role in host antiviral responses and is constitutively expressed in skin cells. RIG-I was found to detect both positive- and negativesense WNV RNA, short cytosolic dsRNA, RNAs with complex secondary structures or $5^{\prime}$-triphosphate RNA, which trigger antiviral immunity (figure 2; [53, 66-68]); sensing of exceeding $2 \mathrm{~kb}$ long dsRNA is more closely related to MDA5 [66]. On the other hand, LGP2, unlike MDA5 and RIG-I, lacks the caspase-recruitment domain (CARD) necessary for recruitment and downstream signalling pathway initiation [69]. It was originally considered to be a negative regulator of RLRs, but the role of LGP2 in positive regulation has also been reported [70, 71]. After activation, MDA5 and RIG-I recruit via their CARD the adaptor molecule interferon promoter-stimulating factor 1 (IPS-1, also called MAVS, for mitochondrial antiviral signalling) leading to the phosphorylation of several transcription factors such as NF- $\mathrm{KB}$, IRF3 and IRF7 (figure 2; [72]). NF- $\mathrm{KB}$ and IRF3 are constitutively expressed in cells, while IRF7 expression is weak in unstimulated cells. These transcription factors are strongly induced after stimuli such as flavivirus infection or type I IFN secretion, leading to the expression of target genes such as type I IFN and ISGs creating an antiviral state to block viral replication [73]. Moreover, type I IFNs activate the JAK-STAT pathways after binding to their receptor (IFN $\alpha$ and $\beta$ receptor, IFNAR) in a paracrine/autocrine way, leading to increased expression of numerous ISGs possessing broad antiviral activities through interference with specific steps in the viral life cycle or regulation of innate immune signalling [74].

\section{TOLL-LIKE RECEPTORS}

Up until now, mainly TLR3 and TLR7 have been implicated in sensing respectively transient dsRNA and ssRNA generated during the flavivirus replicative cycle.

\section{TLR3}

It has been previously described that DENV infection of HUH-7 (hepatocarcinoma cell line) cells resulted in 


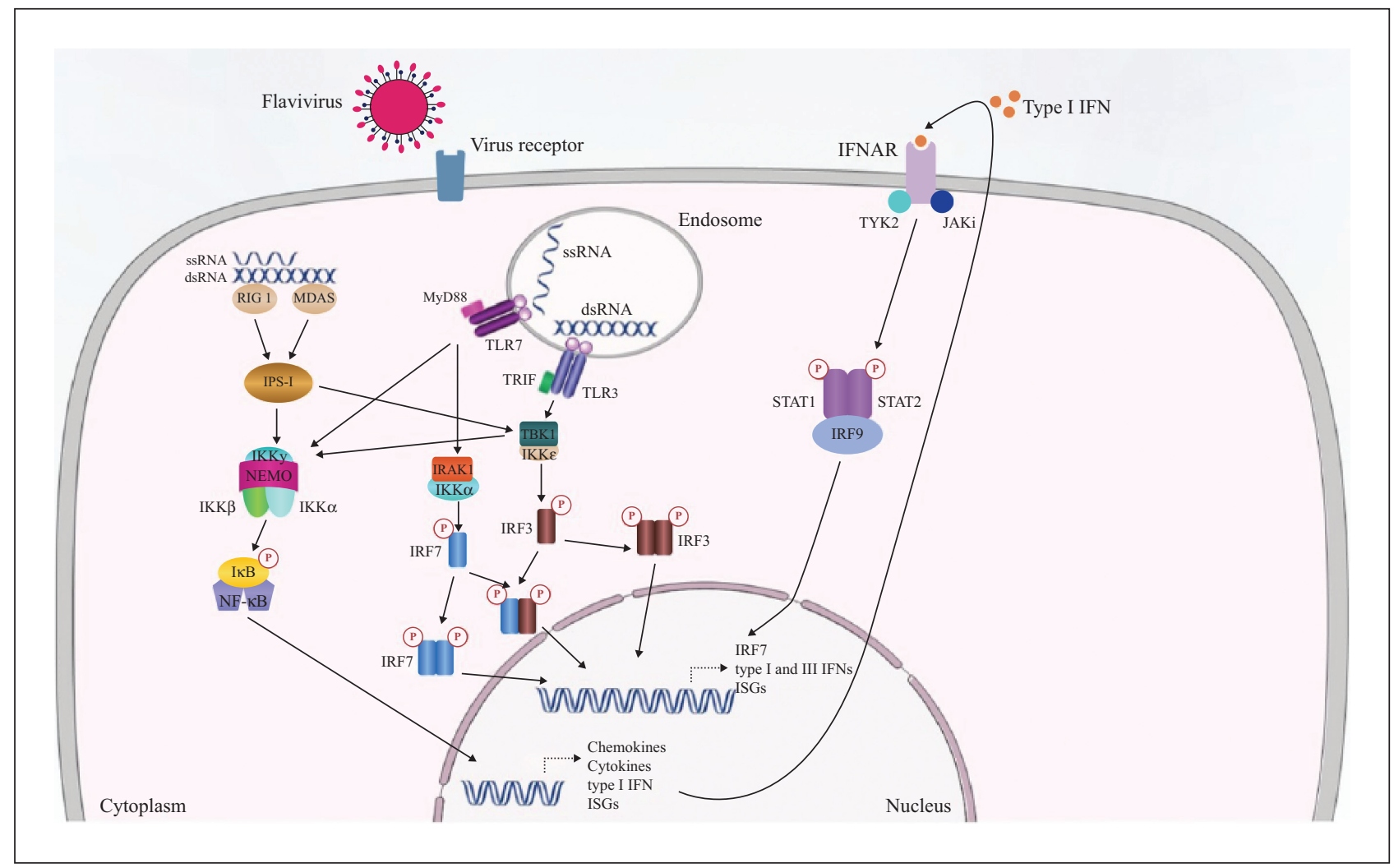

Figure 2

Innate immune response to Dengue (DENV), Zika (ZIKV) and West Nile (WNV) viruses in human skin cells. After viral binding to the host receptor, viral single-stranded (ss) and double-stranded (ds) RNA generated during genome replication can be sensed by host cells' pattern recognition receptors (PRRs). RIG-I can detect both ss and ds RNA whereas MDA5 can detect dsRNA. These cytosolic PRR activation lead to signaling downstream via IPS-1 and NF-кB nuclear translocation contributing to expression of chemokines, cytokines, type I interferon (IFN) and interferon-stimulated genes (ISGs). Extracellular ds and ssRNA can also be detected respectively by Toll-Like Receptor (TLR)3 and TLR7 in the endosomal compartment, which hetero- or homodimerize upon their activation. After recruitment of the adaptor molecule, TIR domain-containing adaptor inducing IFN $\beta$ (TRIF), TLR3 signaling pathway enables nuclear translocation of NF- $\mathrm{B}$, IFN regulatory factor (IRF) 3 and 7 to promote ISGs and type I and III IFN gene expression. TLR7 activation induces MyD88 recruitment and downstream signaling involving IRAK1 or IKK $\alpha$ and IRF7. Finally, in an autocrine (but also paracrine) way, type I IFN binds to IFN $\alpha$ and $\beta$ receptor (IFNAR), amplifying cell antiviral state by promoting ISGs and type I IFN gene expression, including the IRF7 gene.

the upregulation of TLR3 expression and that TLR3 in HEK293 (human embryonic kidney 293) cells recognized DENV2 RNA after endosomal acidification, which resulted in strong IL8 and IFN $\alpha / \beta$ responses [56, 75]. Nasirudeen et al. also demonstrated that downregulation of TLR3 expression by siRNA silencing in HUH7 cells resulted in higher DENV1 replication. Moreover, overexpression of TLR3 in these cells inhibited DENV1 infection through induction of high levels of IFN $\beta$ production [56]. There are, however, few data concerning flaviviral sensing in human skin cells. HDFs and human primary keratinocytes infected with DENV2 and HDFs infected by ZIKV showed an increased level of TLR3 expression $[16,17,46]$. Moreover, it has been shown that inhibition of TLR3 mRNA expression in the human skin fibroblast cell line HFF1 led to a strong increase in the ZIKV RNA load at $48 \mathrm{~h}$ p.i. without modulation of type I IFN mRNA expression [46]. Hence, TLR3 seems to play a key role in ZIKV and DENV sensing.

The role of TLR3 in WNV sensing nonetheless remains controversial. TLR3 deficiency has been described as related to protection against neuro-invasive form of WNV infection in mice highlighted by a reduced viral load, inflammatory response and neuropathology in the brain, while impaired cytokine production and enhanced viral load have been observed in blood; this observation was in comparison with wild-type mice [76]. On the contrary,
Daffis et al. described enhanced WNV replication in the central nervous system of $\mathrm{TLR}^{-/-}$mice after subcutaneous inoculation with only modest differences observed in peripheral viral load level and IFN production [77]. It has also been demonstrated that TLR3 does not modulate WNV replication and IFN induction in vitro in myeloid DCs, macrophages and murine embryonic fibroblasts $[77,78]$. Activation of the IRF3 transcription factor has been described as both dependent and independent of TLR3 [79, 80]. Fredericksen et al. suggested that highly virulent strains of WNV might have evolved to more efficiently stimulate the TLR3-mediated inflammatory response involved in increased blood-brain barrier permeability rather than to disrupt TLR3 signalling [80]. Up until now, no data is available concerning WNV sensing by human skin cells. Unpublished data by our group show that TLR3 is upregulated following primary keratinocyte and dermal fibroblast infection by WNV.

\section{TLR7}

TLR7 is an endosomal receptor involved in the recognition of ssRNA. It has been shown that TLR7 is involved in DENV recognition in plasmacytoid DCs, resulting in type I IFN production inversely proportional to the DENV titre, thereby suggesting an escape mechanism of the virus to immune response $[81,82]$. On the other hand, skin 
fibroblasts have been reported as not expressing TLR7, even following flaviviral infection [17, 46, 83]. Mc Cracken et al. have demonstrated, using an original strategy of mice intradermal inoculation of DENV2 at sites where Aedes aegypti had or had not probed immediately or prior, downregulation of several genes including TLR7 in the presence of mosquito saliva [84]. Consequently, even though TLR7 may be involved in DENV sensing, its role in skin cells remains to be demonstrated.

Concerning WNV, it has been reported that infection of keratinocytes from $\mathrm{TLR}^{-/-}$mice resulted in higher viral replication and weaker expression of IFN $\alpha$, IL1 $\beta$, IL6 and IL12 than in keratinocytes from wild-type mice and that TLR7 response following cutaneous infection promotes LCs migration from the skin to the draining lymph nodes [85]. Nonetheless, in vivo, no difference was observed concerning susceptibility to WNV encephalitis or in the peripheral and brain RNA viral loads between wild-type and TLR $^{-1-}$ mice [85].

\section{RIG-I-like receptors}

MDA5 and RIG-I are induced during HUH-7 cell and MEF infection by DENV1 and 2 respectively, and are involved in IFN $\beta$ production $[56,86]$. Knockdown of these RLRs enhanced cellular permissiveness to DENV1 replication, increased virus propagation and led to higher ISG expression such as of the $2^{\prime}, 5^{\prime}$ oligoadenylate synthetase (OAS)2, ISG15 and ISG56, and to strong activation of IRF3, suggesting that both RIG-I and MDA5 synergistically inhibit DENV replication in vitro $[56,86]$. In HDFs infected by DENV2, type I IFN induction has resulted in an IRF3 nuclear translocation, whereas IRF7 stayed mainly in the cytoplasm. An increased expression of RIG-I, but not MDA5, has been reported [17]. Moreover, the incubation with RIG-I agonist $5^{\prime}$ triphosphorylated RNA prior or after challenge with DENV has been shown to protect against DENV infection by inducing a strong antiviral response via the RIG-I/IPS-1/TBK1/IRF3 signalling pathway [87]. In contrast, during DENV infection of human primary keratinocytes, increased expression of IRF7 transcripts at $48 \mathrm{~h}$ p.i. has been reported, whereas no significant change in IRF3 mRNA expression levels was noted [16]. In addition, several ISGs are induced after keratinocyte infection by DENV such as protein kinase R (PKR), OAS2, Ribonuclease L (RNase L) and RNase L inhibitor (RLI) from $6 \mathrm{~h}$ p.i., highlighting the broad antiviral response of the keratinocytes to flavivirus infection [16]. Indeed, PKR expression is increased after cell priming with type I IFN and binds the dsRNA produced during the viral RNA replication, leading to activation of kinase and inhibition of viral translation and discontinuation of virus spread [88]. OAS2 is involved in viral dsRNA sensing, leading to its cleavage by RNase L, generating smaller dsRNA, which could be detected by MDA5 and RIG-I in order to amplify innate immune response [89].

Cooperation between RIG-I and MDA5 sensing has been described during WNV infection, with viral recognition by these RLRs resulting in establishment of an antiviral response [78, 80]. It has been reported that RIG-I and MDA5 double-knockout mice were more susceptible to WNV infection and that disruption of MDA5 and RIGI pathways abrogated activation of the antiviral response of MEFs to WNV [78, 86, 90]. In addition, RIG-I ${ }^{-1-}$
MEFs showed enhanced viral titres but were still able to mount an innate antiviral response with delayed expression of several genes including IRF3, IRF7, type I IFN, ISG15 and STAT1 [78, 80]. Moreover, IPS-1, an essential RIG-I and MDA5 adaptor molecule, was shown to play a major role in induction of the innate antiviral response of MEFs infected by WNV both in vitro and in vivo, since mice deficient for IPS-1 were more susceptible to WNV infection $[91,92]$. IPS $-1^{-1-}$ mice exhibited higher mortality, higher viral loads in the serum and in the peripheral tissues, earlier brain infection and presented impairment in $\mathrm{T}$ cell expansion, intense inflammatory response and increased $\operatorname{IgM}$ and $\operatorname{IgG}$ production despite weak functionality, suggesting that IPS-1 plays a central role in immunity against WNV, linking innate and adaptive responses [78, 80, 93]. Interestingly, IRF3 is induced only when WNV is replicative in cells and is essential along with IRF7 to control its replication [80, 91, 92]. Other ISGs have been described as displaying significant antiviral activity against WNV in vitro such as C6orf150, DDX24, HPSE, IFI44L, IFI6, IFITM2, IFITM3, IFRD1, IL13RA1, ISG20, MAFK, NAMPT, PAK3, PHF15, SAMD9L, SC4MOL and viperin [94]. Hence, RIG-I and MDA-5 exert a complementary role, whereas RIG-I initiates WNV detection; MDA5 then sustains and enlarges innate immune response. Taken together, the results suggest that WNV and DENV-induced innate immune responses are both RIG-Iand MDA5-dependent [80].

Few data are available concerning skin innate immune sensing of ZIKV. Nonetheless, Hamel et al. have described that infection of primary HDFs with ZIKV resulted in delayed upregulation of RIG-I and MDA5 mRNA as compared with TLR3 expression. Inhibition of TLR3 expression in primary HDFs, unlike that of other PRRs, led to a strong increase of ZIKV RNA copy numbers at $48 \mathrm{~h}$ p.i. but had no consequence on type I IFN mRNA expression in the infected cells. These data suggest that, in primary HDFs, sensing of ZIKV is mainly related to TLR3, which is quickly expressed during infection and relayed by RIG-I and MDA5 with a major role in stemming viral replication [46]. Similar findings have been observed in primary skin fibroblasts infected with DENV in which TLR3 may play a role in the early antiviral response while RIG-I might regulate the amplified response at later time points [17]. Otherwise, human peripheral DCs infected by ZIKV have led to an induction of RIG-I, MDA5 and STAT1 and -2, and ISGs such as viperin and IFIT-1, -2 and -3 expression [47]. Interestingly, ZIKV replication is restricted by RIG-I agonists but not by type I IFN [47].

Other proteins involved in flavivirus sensing such as LGP2 can contribute to cytosolic RNA sensing, but its role in innate immunity remains unclear [95]. It has been reported that LGP2 was not essential for induction of innate immunity but was indeed required for controlling antigenspecific $\mathrm{CD}^{+} \mathrm{T}$ cell activation, survival and fitness during peripheral $\mathrm{T}$ cell-number expansion in response to WNV infection, independently of MDA5 [94, 96]. In addition, LGP2 mRNA expression is induced after ZIKV infection of human DCs [47].

Recently a cytosolic DNA sensor, cyclic GMP-AMP synthase (cGAS, also known as MB21D1) signalling through downstream signalling molecule STING to induce type I IFN production [97], was described as being involved in 
inhibition of replication of several RNA viruses such as WNV in a RIG-I-dependent manner and suggested as being important for basal expression of ISGs [98]. Schoggins et al. demonstrated that ectopic expression of cGAS inhibited WNV replication and that $\mathrm{cGAS}^{-1-}$ mice exhibited increased lethality after WNV infection [99]. The mechanism by which cGAS becomes activated following WNV infection remains unknown.

Few data have been published concerning the role of inflammasome during infections by flaviviruses. Inflammasome is a multiprotein complex belonging to the innate immunity system. Inflammasome activation is a consequence of host cell's cytosolic NLRs such as NLRP (nucleotidic domain and leucine-rich repeat containing (NLR) protein)-1 and -3, NLRC4 (nlr family, caspase recruitment domain-containing 4) and AIM2 (absent in melanoma) having sensed "danger signals", and it leads to IL1 $\beta$, IL18 and IL33 pro-inflammatory cytokine secretion [100]. NLRP3 inflammasome is activated following WNV and DENV infection; these infections in humans are associated with elevated levels of systemic IL1 $\beta$ [101, 102]. ZIKV has been shown to be responsible for activation of the inflammasome pathway in fibroblasts, since AIM2, which is a DNA sensor involved in caspase 1 activation, and IL1 $\beta$ mRNA were strongly induced after infection [46, 103].

\section{VIRUS EVASION FROM INNATE IMMUNITY}

Following virus inoculation in the skin, a strong innate immune antiviral response is quickly elicited, both in infected and in uninfected neighbouring cells to dam up virus infection and dissemination. Nonetheless, various mechanisms of immune evasion have been elaborated by DENV and WNV to counteract host defenses. Flaviviruses can interfere with innate immune response at three distinct and complementary levels: (i) hiding or modifying viral RNA to evade immune sensing; (ii) directly interacting with PRRs to inhibit their functions; and (iii) antagonizing key signalling proteins downstream from PRRs [104]. Firstly, flavivirus can escape RNA detection by cytosolic PRRs by collecting cell membrane to define replication compartments designated as viral replication factories and effectively isolated from the rest of the cell cytoplasm $[105,106]$. They can also modify the $5^{\prime}$ end of their genome, via the viral 2'-O-methyltransferase NS5, with a type 1 cap structure identical to that of cellular mRNA, thereby avoiding "non-self" host detection [107, 108]. Secondly, they can subvert innate immune response by producing sub-genomic flavivirus (sf) RNA, which are small RNAs around 500 nucleotides derived from the $3^{\prime}$ untranslated region and generated from incomplete degradation of genomic RNA by the $5^{\prime}-3^{\prime}$ exoribonuclease, XRN1 [109]. These sfRNAs are necessary for effective viral growth and pathogenicity in cell culture and in mice [110]. They have been recently reported to play a modulatory role in host antiviral response as RIG-I and MDA5 agonists [111, 112]. Thirdly, non-structural flavivirus proteins such as the NS2B/3 protein, which functions as serine protease, have been shown to block PRRs and JAK/STAT downstream signalling pathways through degradation, inhibition of phosphorylation and nuclear translocation of signal proteins such as STAT1, STAT2 or IRF3 [47, 113].
In addition, the vector can contribute to immune escape. When probing, the mosquito vector not only inoculates virus, but also saliva into the host's skin, primarily in the extravascular space [114]. Proteins in mosquito saliva can alter innate antiviral immune responses, thereby decreasing cytokine levels and disturbing other components of innate immunity which leads to local immunosuppression or dysregulation, and enhanced spread and replication of the virus [16, 115-119]. Indeed, Surasombatpattana et al. demonstrated that proteins of Aedes saliva modulate human primary keratinocyte innate immune response to DENV by reducing mRNA expression of IFN $\alpha$, IFN $\beta$, IRF3, IRF7 and AMPs such as LL37, RNase 7 or S100A7 $[115,120]$.

\section{CONCLUSION}

Virus inoculation in the skin is a key step in the pathophysiology of flavivirus infection, since this complex organ contains numerous permissive cells to ZIKV, DENV and WNV, thereby constituting not only the first site of viral replication in the host but also the initiation site of the immune response. Indeed, skin cells harbour a wide range of innate immunity receptors involved in both extra- and intracellular viral sensing, leading to quick induction of an antiviral state in infected as well as in uninfected neighbouring cells, thereby limiting viral spread. Further characterization of the skin's antiviral effector molecules and their precise role in early antiviral response will provide greater insights into host-arbovirus interactions, why these viruses can establish an infection and how it will be possible to tilt the balance in favour of the host at their first point of entry and initial replication site, well before systemic spread occurs to the target organs, such as the central nervous system, the liver or the foetus, and also before the adaptive immune response kicks in.

Acknowledgments. We thank Jeffrey Arsham for the English revision of the paper.

Disclosure. Financial support: none. Conflict of interest: none.

\section{REFERENCES}

1. Brown C. Zika virus outbreaks in Asia and South America. Can Med Assoc J 2016; 188: E34.

2. Krow-Lucal E, Lindsey NP, Lehman J, Fischer M, Staples JE. West Nile virus and other nationally notifiable arboviral diseases, United States, 2015. Morb Mortal Wkly Rep 2017; 66: 51-5.

3. Schneider BS, Soong L, Girard YA, Campbell G, Mason P, Higgs S. Potentiation of West Nile encephalitis by mosquito feeding. Viral Immunol 2006; 19: 74-82.

4. Brown AN, Kent KA, Bennett CJ, Bernard KA. Tissue tropism and neuroinvasion of West Nile virus do not differ for two mouse strains with different survival rates. Virology 2007; 368: 422-30.

5. Briant L, Desprès $\mathrm{P}$, Choumet V, Missé D. Role of skin immune cells on the host susceptibility to mosquito-borne viruses. Virology 2014; 464-465: 26-32.

6. Nestle FO, Di Meglio P, Qin J-Z, Nickoloff BJ. Skin immune sentinels in health and disease. Nat Rev Immunol 2009; 9: 679-91. 
7. Kubo A, Nagao K, Yokouchi M, Sasaki H, Amagai M. External antigen uptake by Langerhans cells with reorganization of epidermal tight junction barriers. J Exp Med 2009; 206: 2937-46.

8. Seneschal J, Clark RA, Gehad A, Baecher-Allan CM, Kupper TS. Human epidermal Langerhans cells maintain immune homeostasis in skin by activating skin resident regulatory T cells. Immunity 2012; 36: 873-84

9. WHO. Scientific working group report on Dengue. Geneva: WHO, 2008.

10. Simmons CP, Farrar JJ, Nguyen van VC, Wills B. Dengue. N Engl J Med 2012; 366: 1423-32.

11. Wu SJ, Grouard-Vogel G, Sun W, et al. Human skin Langerhans cells are targets of Dengue virus infection. Nat Med 2000; 6: 816-20.

12. Limon-Flores AY, Perez-Tapia M, Estrada-Garcia I, et al. Dengue virus inoculation to human skin explants: an effective approach to assess in situ the early infection and the effects on cutaneous dendritic cells. Int J Exp Pathol 2005; 86: 323-34.

13. Schmid MA, Harris E. Monocyte recruitment to the dermis and differentiation to dendritic cells increases the targets for Dengue virus replication. PLoS Pathog 2014; 10: e1004541.

14. Taweechaisupapong S, Sriurairatana S, Angsubhakorn S, Yoksan $\mathrm{S}$, Bhamarapravati $\mathrm{N}$. In vivo and in vitro studies on the morphological change in the monkey epidermal Langerhans cells following exposure to Dengue 2 (16681) virus. Southeast Asian J Trop Med Public Health 1996; 27: 664-72.

15. Cerny D, Haniffa M, Shin A, et al. Selective susceptibility of human skin antigen presenting cells to productive Dengue virus infection. PLoS Pathog 2014; 10: e1004548.

16. Surasombatpattana $\mathrm{P}$, Hamel R, Patramool S, et al. Dengue virus replication in infected human keratinocytes leads to activation of antiviral innate immune responses. Infect Genet Evol 2011; 11 : 1664-73.

17. Bustos-Arriaga J, García-Machorro J, León-Juárez $\mathrm{M}$, et al. Activation of the innate immune response against DENV in normal non-transformed human fibroblasts. PLoS Negl Trop Dis 2011; 5: e1420.

18. Bustos-Arriaga J, Mita-Mendoza NK, Lopez-Gonzalez M, et al. Soluble mediators produced by the crosstalk between microvascular endothelial cells and Dengue-infected primary dermal fibroblasts inhibit Dengue virus replication and increase leukocyte transmigration. Immunol Res 2016; 64: 392-403.

19. Kurane I, Janus J, Ennis FA. Dengue virus infection of human skin fibroblasts in vitro production of IFN-beta, IL-6 and GM-CSF. Arch Virol 1992; 124: 21-30.

20. St John AL, Rathore APS, Raghavan B, Ng M-L, Abraham SN. Contributions of mast cells and vasoactive products, leukotrienes and chymase, to Dengue virus-induced vascular leakage. eLife 2013; 2: e00481.

21. Chu Y-T, Wan S-W, Anderson R, Lin Y-S. Mast cell-macrophage dynamics in modulation of Dengue virus infection in skin. Immunology 2015; 146: 163-72.

22. Troupin A, Shirley D, Londono-Renteria B, et al. A role for human skin mast cells in Dengue virus infection and systemic spread. $J$ Immunol 2016; 197: 382-4391.

23. Dauphin G, Zientara S, Zeller H, Murgue B. West Nile: worldwide current situation in animals and humans. Comp Immunol Microbiol Infect Dis 2004; 27: 343-55.

24. Di Sabatino D, Bruno R, Sauro F, et al. Epidemiology of West Nile disease in Europe and in the Mediterranean Basin from 2009 to 2013. Biomed Res Int 2014; 2014: 907852.
25. O'Leary DR, Marfin AA, Montgomery SP, et al. The epidemic of West Nile virus in the United States, 2002. Vector Borne Zoonotic Dis 2004; 4: 61-70.

26. Lim P-Y, Behr MJ, Chadwick CM, Shi P-Y, Bernard KA. Keratinocytes are cell targets of West Nile virus in vivo. J Virol 2011; 85: 5197-201.

27. Nemeth N, Young G, Ndaluka C, Bielefeldt-Ohmann H, Komar N, Bowen R. Persistent West Nile virus infection in the house sparrow (Passer domesticus). Arch Virol 2009; 154: 783-9.

28. Appler KK, Brown AN, Stewart BS, et al. Persistence of West Nile virus in the central nervous system and periphery of mice. PloS One 2010; 5: e10649.

29. Arnold SJ, Osvath SR, Hall RA, King NJC, Sedger LM. Regulation of antigen processing and presentation molecules in West Nile virus-infected human skin fibroblasts. Virology 2004; 324: 286-96.

30. Hoover LI, Fredericksen BL. IFN-dependent and -independent reduction in West Nile virus infectivity in human dermal fibroblasts. Viruses 2014; 6: 1424-41.

31. Davis CW, Nguyen H-Y, Hanna SL, Sánchez MD, Doms RW, Pierson TC. West Nile virus discriminates between DC-SIGN and DC-SIGNR for cellular attachment and infection. $J$ Virol 2006; 80: 1290-301

32. Qian F, Wang X, Zhang L, et al. Impaired interferon signaling in dendritic cells from older donors infected in vitro with West Nile virus. J Infect Dis 2011; 203: 1415-24.

33. Martina BEE, Koraka P, van den Doel P, Rimmelzwaan GF, Haagmans BL, Osterhaus ADME. DC-SIGN enhances infection of cells with glycosylated West Nile virus in vitro and virus replication in human dendritic cells induces production of IFN-alpha and TNFalpha. Virus Res 2008; 135: 64-71.

34. Silva MC, Guerrero-Plata A, Gilfoy FD, Garofalo RP, Mason PW. Differential activation of human monocyte-derived and plasmacytoid dendritic cells by West Nile virus generated in different host cells. J Virol 2007; 81: 13640-8

35. Rawle DJ, Setoh YX, Edmonds JH, Khromykh AA. Comparison of attenuated and virulent West Nile virus strains in human monocytederived dendritic cells as a model of initial human infection. $J$ Virol $2015 ; 12: 46$.

36. Kovats S, Turner S, Simmons A, Powe T, Chakravarty E, AlberolaIla J. West Nile virus-infected human dendritic cells fail to fully activate invariant natural killer T cells. Clin Exp Immunol 2016; 186: 214-26.

37. Tupin E, Kinjo Y, Kronenberg M. The unique role of natural killer $\mathrm{T}$ cells in the response to microorganisms. Nat Rev Microbiol 2007; 5: 405-17.

38. Brennan PJ, Brigl M, Brenner MB. Invariant natural killer T cells: an innate activation scheme linked to diverse effector functions. Nat Rev Immunol 2013; 13: 101-17.

39. Schmid ET, Pang IK, Carrera Silva EA, et al. AXL receptor tyrosine kinase is required for $\mathrm{T}$ cell priming and antiviral immunity. eLife $2016 ; 5$.

40. Johnston LJ, Halliday GM, King NJ. Phenotypic changes in Langerhans cells after infection with arboviruses: a role in the immune response to epidermally acquired viral infection? J Virol 1996; 70: 4761-6

41. Johnston LJ, Halliday GM, King NJ. Langerhans cells migrate to local lymph nodes following cutaneous infection with an arbovirus. J Invest Dermatol 2000; 114: 560-8.

42. Cumberbatch M, Dearman RJ, Kimber I. Langerhans cells require signals from both tumour necrosis factor-alpha and interleukin-1 beta for migration. Immunology 1997; 92: 388-95. 
43. Byrne SN, Halliday GM, Johnston LJ, King NJ. Interleukin-1beta but not tumor necrosis factor is involved in West Nile virus-induced Langerhans cell migration from the skin in C57BL/6 mice. J Invest Dermatol 2001; 117: 702-9.

44. Mlakar J, Korva M, Tul N, et al. Zika virus associated with microcephaly. N Engl J Med 2016; 374: 951-8.

45. Haddow AD, Schuh AJ, Yasuda CY, et al. Genetic characterization of Zika virus strains: geographic expansion of the Asian lineage. PLoS Negl Trop Dis 2012; 6: e1477.

46. Hamel R, Dejarnac O, Wichit S, et al. Biology of Zika virus infection in human skin cells. $J$ Virol 2015; 89: 8880-96.

47. Bowen JR, Quicke KM, Maddur MS, et al. Zika virus antagonizes type I interferon responses during infection of human dendritic cells. PLoS Pathog 2017; 13: e1006164.

48. Proost P, Vynckier A-K, Mahieu F, Van Damme J. Microbial Toll-like receptor ligands differentially regulate CXCL10/IP-10 expression in fibroblasts and mononuclear leukocytes in synergy with IFN-gamma and provide a mechanism for enhanced synovial chemokine levels in septic arthritis. Eur J Immunol 2003; 33: 3146-53.

49. Tamagawa-Mineoka R. Important roles of platelets as immune cells in the skin. J Dermatol Sci 2015; 77: 93-101.

50. Gasque P, Jaffar-Bandjee MC. The immunology and inflammatory responses of human melanocytes in infectious diseases. $J$ Infect 2015; 71:413-21.

51. Brinton MA. Replication cycle and molecular biology of the West Nile virus. Viruses 2013; 6: 13-53.

52. Perera-Lecoin M, Meertens L, Carnec X, Amara A. Flavivirus entry receptors: an update. Viruses 2013; 6: 69-88.

53. Shipley JG, Vandergaast R, Deng L, Mariuzza RA, Fredericksen BL. Identification of multiple RIG-I-specific pathogen associated molecular patterns within the West Nile virus genome and antigenome. Virology 2012; 432: 232-8.

54. Westaway EG, Khromykh AA, Mackenzie JM. Nascent flavivirus RNA colocalized in situ with double-stranded RNA in stable replication complexes. Virology 1999; 258: 108-17.

55. Chambers TJ, Hahn CS, Galler R, Rice CM. Flavivirus genome organization, expression, and replication. Апnи Rev Microbiol 1990; 44: 649-88.

56. Nasirudeen AMA, Wong HH, Thien $\mathrm{P}, \mathrm{Xu} \mathrm{S}$, Lam K-P, Liu DX. RIG-I, MDA5 and TLR3 synergistically play an important role in restriction of Dengue virus infection. PLoS Negl Trop Dis 2011; 5: e926

57. Lebre MC, van der Aar AMG, van Baarsen L, et al. Human keratinocytes express functional Toll-like receptor $3,4,5$, and $9 . J$ Invest Dermatol 2007; 127: 331-41.

58. Renn CN, Sanchez DJ, Ochoa MT, et al. TLR activation of Langerhans cell-like dendritic cells triggers an antiviral immune response. J Immunol 2006; 177: 298-305.

59. Yu N, Zhang S, Zuo F, Kang K, Guan M, Xiang L. Cultured human melanocytes express functional toll-like receptors 2-4, 7 and 9. $J$ Dermatol Sci 2009; 56: 113-20.

60. Kulka M, Fukuishi N, Rottem M, Mekori YA, Metcalfe DD. Mast cells, which interact with Escherichia coli, up-regulate genes associated with innate immunity and become less responsive to Fc(epsilon)RI-mediated activation. JLeukoc Biol 2006; 79: 339-50.

61. Zhang FX, Kirschning CJ, Mancinelli R, et al. Bacterial lipopolysaccharide activates nuclear factor-kappaB through interleukin-1 signaling mediators in cultured human dermal endothelial cells and mononuclear phagocytes. J Biol Chem 1999; 274 : 7611-4.

62. Faure E, Equils O, Sieling PA, et al. Bacterial lipopolysaccharide activates NF-kappaB through toll-like receptor 4 (TLR-4) in cultured human dermal endothelial cells. Differential expression of TLR-4 and TLR-2 in endothelial cells. J Biol Chem 2000; 275: 11058-63.

63. Medzhitov R. Toll-like receptors and innate immunity. Nat Rev Immunol 2001; 1: 135-45.

64. Akira S. TLR signaling. Curr Top Microbiol Immunol 2006; 311: $1-16$.

65. Lester SN, Li K. Toll-like receptors in antiviral innate immunity. $J$ Mol Biol 2014; 426: 1246-64.

66. Kato H, Takeuchi O, Sato S, et al. Differential roles of MDA5 and RIG-I helicases in the recognition of RNA viruses. Nature 2006; 441: 101-5

67. Pichlmair A, Schulz O, Tan CP, et al. RIG-I-mediated antiviral responses to single-stranded RNA bearing $5^{\prime}$-phosphates. Science 2006; 314: 997-1001.

68. Schlee M, Roth A, Hornung V, et al. Recognition of $5^{\prime}$ triphosphate by RIG-I helicase requires short blunt double-stranded RNA as contained in panhandle of negative-strand virus. Immunity 2009; 31: 25-34.

69. Bruns AM, Pollpeter D, Hadizadeh N, Myong S, Marko JF, Horvath CM. ATP hydrolysis enhances RNA recognition and antiviral signal transduction by the innate immune sensor, laboratory of genetics and physiology 2 (LGP2). J Biol Chem 2013; 288: 938-46.

70. Rothenfusser S, Goutagny N, DiPerna G, et al. The RNA helicase Lgp2 inhibits TLR-independent sensing of viral replication by retinoic acid-inducible gene-I. J Immunol 2005; 175: 5260-8.

71. Satoh T, Kato H, Kumagai Y, et al. LGP2 is a positive regulator of RIG-I- and MDA5-mediated antiviral responses. Proc Natl Acad Sci U S A 2010; 107: 1512-7.

72. Sato M, Suemori H, Hata N, et al. Distinct and essential roles of transcription factors IRF-3 and IRF-7 in response to viruses for IFN-alpha/beta gene induction. Immunity 2000; 13: 539-48.

73. Kawai T, Akira S. Toll-like receptor and RIG-I-like receptor signaling. Ann N Y Acad Sci 2008; 1143: 1-20.

74. Suthar MS, Aguirre S, Fernandez-Sesma A. Innate immune sensing of flaviviruses. PLoS Pathog 2013; 9: e1003541.

75. Tsai Y-T, Chang S-Y, Lee C-N, Kao C-L. Human TLR3 recognizes Dengue virus and modulates viral replication in vitro. Cell Microbiol 2009; 11: 604-15.

76. Wang T, Town T, Alexopoulou L, Anderson JF, Fikrig E, Flavell RA. Toll-like receptor 3 mediates West Nile virus entry into the brain causing lethal encephalitis. Nat Med 2004; 10: 1366-73.

77. Daffis S, Samuel MA, Suthar MS, Gale M, Diamond MS. Toll-like receptor 3 has a protective role against West Nile virus infection. $J$ Virol 2008; 82: 10349-58.

78. Fredericksen BL, Keller BC, Fornek J, Katze MG, Gale M. Establishment and maintenance of the innate antiviral response to West Nile virus involves both RIG-I and MDA5 signaling through IPS-1. J Virol 2008; 82: 609-16.

79. Fredericksen BL, Smith M, Katze MG, Shi P-Y, Gale M. The host response to West Nile virus infection limits viral spread through the activation of the interferon regulatory factor 3 pathway. $J$ Virol 2004; 78: 7737-47

80. Fredericksen BL, Gale M. West Nile virus evades activation of interferon regulatory factor 3 through RIG-I-dependent 
and -independent pathways without antagonizing host defense signaling. J Virol 2006; 80: 2913-23.

81. Wang JP, Liu P, Latz E, Golenbock DT, Finberg RW, Libraty DH. Flavivirus activation of plasmacytoid dendritic cells delineates key elements of TLR7 signaling beyond endosomal recognition. $J$ Immunol 2006; 177: 7114-21.

82. Sun P, Fernandez S, Marovich MA, et al. Functional characterization of ex vivo blood myeloid and plasmacytoid dendritic cells after infection with Dengue virus. Virology 2009; 383: 207-15.

83. Paladino P, Cummings DT, Noyce RS, Mossman KL. The IFNindependent response to virus particle entry provides a first line of antiviral defense that is independent of TLRs and retinoic acidinducible gene I. J Immunol 2006; 177: 8008-16.

84. McCracken MK, Christofferson RC, Chisenhall DM, Mores CN. Analysis of early Dengue virus infection in mice as modulated by Aedes aegypti probing. J Virol 2014; 88: 1881-9.

85. Welte T, Reagan K, Fang H, et al. Toll-like receptor 7-induced immune response to cutaneous West Nile virus infection. J Gen Virol 2009; 90: 2660-8.

86. Loo Y-M, Fornek J, Crochet N, et al. Distinct RIG-I and MDA5 signaling by RNA viruses in innate immunity. J Virol 2008; 82: 335-45.

87. Olagnier D, Scholte FEM, Chiang C, et al. Inhibition of Dengue and chikungunya virus infections by RIG-I-mediated type I interferonindependent stimulation of the innate antiviral response. $J$ Virol 2014; 88: 4180-94.

88. Balachandran S, Roberts PC, Brown LE, et al. Essential role for the dsRNA-dependent protein kinase PKR in innate immunity to viral infection. Immunity 2000; 13: 129-41.

89. Sadler AJ, Williams BRG. Interferon-inducible antiviral effectors. Nat Rev Immunol 2008; 8: 559-68.

90. Errett JS, Suthar MS, McMillan A, Diamond MS, Gale M. The essential, nonredundant roles of RIG-I and MDA5 in detecting and controlling West Nile virus infection. J Virol 2013; 87: 11416-25.

91. Daffis S, Samuel MA, Keller BC, Gale M, Diamond MS. Cellspecific IRF-3 responses protect against West Nile virus infection by interferon-dependent and -independent mechanisms. PLoS Pathog 2007; 3: e106.

92. Daffis S, Suthar MS, Szretter KJ, Gale M, Diamond MS. Induction of IFN-beta and the innate antiviral response in myeloid cells occurs through an IPS-1-dependent signal that does not require IRF-3 and IRF-7. PLoS Pathog 2009; 5: e1000607.

93. Suthar MS, Ma DY, Thomas S, et al. IPS-1 is essential for the control of West Nile virus infection and immunity. PLoS Pathog 2010; 6: e1000757.

94. Lazear HM, Diamond MS. New insights into innate immune restriction of West Nile virus infection. Curr Opin Virol 2015; 11: 1-6.

95. Goubau D, Deddouche S, Reis e Sousa C. Cytosolic sensing of viruses. Immunity 2013; 38: 855-69.

96. Suthar MS, Ramos HJ, Brassil MM, et al. The RIG-I-like receptor LGP2 controls CD8(+) T cell survival and fitness. Immunity 2012; $37: 235-48$

97. Cai X, Chiu Y-H, Chen ZJ. The cGAS-cGAMP-STING pathway of cytosolic DNA sensing and signaling. Mol Cell 2014; 54: 289-96.

98. Schoggins JW. Interferon-stimulated genes: roles in viral pathogenesis. Curr Opin Virol 2014; 6: 40-6.

99. Schoggins JW, MacDuff DA, Imanaka N, Gainey MD, Shrestha B, Eitson JL, et al. Pan-viral specificity of IFN-induced genes reveals new roles for cGAS in innate immunity. Nature 2014; 505: 691-5.
100. Lamkanfi M, Dixit VM. Modulation of inflammasome pathways by bacterial and viral pathogens. J Immunol 2011; 187: 597-602.

101. Ramos HJ, Lanteri MC, Blahnik G, et al. IL-1 $\beta$ signaling promotes CNS-intrinsic immune control of West Nile virus infection. PLoS Pathog 2012; 8: e1003039.

102. Bozza FA, Cruz OG, Zagne SMO, et al. Multiplex cytokine profile from Dengue patients: MIP-1beta and IFN-gamma as predictive factors for severity. BMC Infect Dis 2008; 8: 86.

103. Hornung V, Ablasser A, Charrel-Dennis M, et al. AIM2 recognizes cytosolic dsDNA and forms a caspase-1-activating inflammasome with ASC. Nature 2009; 458: 514-8.

104. Gack MU, Diamond MS. Innate immune escape by Dengue and West Nile viruses. Curr Opin Virol 2016; 20: 119-28.

105. Gillespie LK, Hoenen A, Morgan G, Mackenzie JM. The endoplasmic reticulum provides the membrane platform for biogenesis of the flavivirus replication complex. J Virol 2010; 84: 10438-47.

106. den Boon JA, Ahlquist P. Organelle-like membrane compartmentalization of positive-strand RNA virus replication factories. Annu Rev Microbiol 2010; 64: 241-56.

107. Daffis S, Szretter KJ, Schriewer J, et al. 2'-O methylation of the viral mRNA cap evades host restriction by IFIT family members. Nature 2010; 468: 452-6.

108. Szretter KJ, Daniels BP, Cho H, et al. $2^{\prime}-\mathrm{O}$ methylation of the viral mRNA cap by West Nile virus evades ifit1-dependent and -independent mechanisms of host restriction in vivo. PLoS Pathog 2012; 8: e1002698.

109. Clarke BD, Roby JA, Slonchak A, Khromykh AA. Functional noncoding RNAs derived from the flavivirus $3^{\prime}$ untranslated region. Virus Res 2015; 206: 53-61.

110. Pijlman GP, Funk A, Kondratieva N, et al. A highly structured, nuclease-resistant, noncoding RNA produced by flaviviruses is required for pathogenicity. Cell Host Microbe 2008; 4: 579-91.

111. Schuessler A, Funk A, Lazear HM, et al. West Nile virus noncoding subgenomic RNA contributes to viral evasion of the type I interferon-mediated antiviral response. J Virol 2012; 86: 5708-18.

112. Manokaran G, Finol E, Wang C, et al. Dengue subgenomic RNA binds TRIM25 to inhibit interferon expression for epidemiological fitness. Science 2015: 350217-21.

113. Angleró-Rodríguez YI, Pantoja P, Sariol CA. Dengue virus subverts the interferon induction pathway via NS2B/3 protease-ІкB kinase epsilon interaction. Clin Vaccine Immunol 2014; 21: 29-38.

114. Styer LM, Kent KA, Albright RG, Bennett CJ, Kramer LD, Bernard KA. Mosquitoes inoculate high doses of West Nile virus as they probe and feed on live hosts. PLoS Pathog 2007; 3: 1262-70.

115. Surasombatpattana P, Ekchariyawat P, Hamel R, et al. Aedes aegypti saliva contains a prominent $34-\mathrm{kDa}$ protein that strongly enhances Dengue virus replication in human keratinocytes. J Invest Dermatol 2014; 134: 281-4

116. Fontaine A, Diouf I, Bakkali N, et al. Implication of haematophagous arthropod salivary proteins in host-vector interactions. Parasit Vectors 2011; 4: 187.

117. Moser LA, Lim P-Y, Styer LM, Kramer LD, Bernard KA. Parameters of mosquito-enhanced West Nile virus infection. $J$ Virol 2015; 90: 292-9.

118. Schmid MA, Glasner DR, Shah S, Michlmayr D, Kramer LD, Harris E. Mosquito saliva increases endothelial permeability in the skin, immune cell migration, and Dengue pathogenesis during antibodydependent enhancement. PLoS Pathog 2016; 12: e1005676. 
119. Schneider BS, Soong L, Coffey LL, Stevenson HL, McGee CE, Higgs S. Aedes aegypti saliva alters leukocyte recruitment and cytokine signaling by antigen-presenting cells during West Nile virus infection. PloS One 2010; 5: e11704.
120. Surasombatpattana P, Patramool S, Luplertlop N, Yssel H, Missé D. Aedes aegypti saliva enhances Dengue virus infection of human keratinocytes by suppressing innate immune responses. $J$ Invest Dermatol 2012; 132: 2103-5. 